\title{
ASK1/2 signaling promotes inflammation in a mouse model of neutrophilic dermatosis
}

\author{
Sarang Tartey, ${ }^{1}$ Prajwal Gurung, ${ }^{1,2}$ Tejasvi Krishna Dasari, ${ }^{1}$ Amanda Burton, ${ }^{1}$ and Thirumala-Devi Kanneganti ${ }^{1}$ \\ 'Department of Immunology, St. Jude Children's Research Hospital, Memphis, Tennessee, USA. ${ }^{2}$ Inflammation Program, University of lowa, Iowa City, lowa, USA.
}

\begin{abstract}
Mice homozygous for the Tyr208Asn amino acid substitution in the carboxy terminus of Src homology region 2 (SH2) domain-containing phosphatase 1 (SHP-1) (referred to as Ptpn6 ${ }^{\text {spin }}$ mice) spontaneously develop a severe inflammatory disease resembling neutrophilic dermatosis in humans. Disease in Ptpn6 ${ }^{\text {sin }}$ mice is characterized by persistent footpad swelling and suppurative inflammation. Recently, in addition to IL-1 $\alpha$ and IL-1R signaling, we demonstrated a pivotal role for several kinases such as SYK, RIPK1, and TAK1 in promoting inflammatory disease in Ptpn6 ${ }^{\text {spin }}$ mice. In order to identify new kinases involved in SHP-1-mediated inflammation, we took a genetic approach and discovered apoptosis signal-regulating kinases 1 and 2 (ASK1 and ASK2) as novel kinases regulating Ptpn6-mediated footpad inflammation. Double deletion of ASK1 and ASK2 abrogated cutaneous inflammatory disease in Ptpn6 ${ }^{\text {spin }}$ mice. This double deletion further rescued the splenomegaly and lymphomegaly caused by excessive neutrophil infiltration in Ptpn $6^{\text {spin }}$ mice. Mechanistically, ASK regulates Ptpn $^{\text {spin }}$-mediated disease by controlling proinflammatory signaling in the neutrophils. Collectively, the present study identifies SHP-1 and ASK signaling crosstalk as a critical regulator of IL-1 $\alpha$-driven inflammation and opens future avenues for finding novel drug targets to treat neutrophilic dermatosis in humans.
\end{abstract}

\section{Introduction}

Mutations in the PTPN6 gene that encodes for the protein tyrosine phosphatase Src homology region 2 (SH2) domain-containing phosphatase 1 (SHP-1) have been linked with autoinflammatory and autoimmune diseases in humans (1-3). Hypomorphic Ptpn6 mutant mice with a homozygous Tyr208Asn amino acid mutation (exhibiting spontaneous inflammation, or spin, and referred to as $P \operatorname{tpn} 6^{\text {spin }}$ mice here) develop persistent footpad swelling and suppurative inflammation that are very similar to neutrophilic dermatosis in humans $(4,5)$. Neutrophilic dermatosis encompasses disorders that are characterized by neutrophilic infiltration not associated with infection (6), such as Sweet's Syndrome and pyoderma gangrenosum. While the essential role of IL-1 in promoting these autoinflammatory diseases is well established, the specific roles for IL- $1 \alpha$ and IL- $1 \beta$ have only recently been recognized $(5,7)$. Using Ptpn $6^{\text {spin }}$ mice as a model of inflammatory disease, we previously showed that IL- $1 \alpha$, but not IL- $1 \beta$, is a central cytokine that promotes neutrophilic footpad inflammation (5). Mechanistically, receptor interacting protein kinase 1 (RIPK1) has been shown to regulate IL-1 $\alpha$ expression independently of RIPK3, suggesting a role for a RIPK1 and IL-1 $\alpha$ signaling axis in driving footpad inflammation (5). Several studies including ours have demonstrated that hematopoietic or neutrophil-specific deletion of Ptpn6 is sufficient to promote neutrophilic footpad inflammation $(5,8)$.

Genetic approaches enabled us to delineate the molecular mechanisms and signaling pathways that are regulated by SHP-1 to modulate inflammation. Our recent studies demonstrated

Conflict of interest: The authors have declared that no conflict of interest exists. Submitted: November 3, 2017; Accepted: February 27, 2018.

Reference information: J Clin Invest. 2018;128(5):2042-2047.

https://doi.org/10.1172/JCI98446. a central role for IL-1 $\alpha$, IL-1R, MyD88, tumor growth factor $\beta$-activated kinase 1 (TAK1), spleen tyrosine kinase (SYK), and RIPK1 in promoting inflammatory disease in Ptpn $^{\text {spin }}$ mice, independent of interferon- $\alpha / \beta$ receptor (IFNAR), stimulator of interferon genes (STING), integrin beta 3 (ITGB3), and nucleotidebinding oligomerization domain 2-RIPK2 (NOD2-RIPK2) signaling $(5,9,10)$. Excessive inflammatory responses and persistent tissue damage in $P \operatorname{tpn} 6^{\text {spin }}$ mice are driven by RIPK1-mediated MAPKs and NF- $\kappa \mathrm{B}$ signaling in hematopoietic cells $(5,9)$. Given that TAK1 is a central regulator of NF- $\mathrm{BB}, \mathrm{ERK}$, and p38 downstream of TLR activation (11-13), we hypothesized that other MAPKs might also play a pivotal role in mediating inflammatory skin disease in Ptpn $6^{\text {spin }}$ mice.

MAPKs are Ser/Thr kinases involved in relaying extracellular signals to regulate survival, apoptosis, metabolism, inflammation, and many other cellular processes (14). Conventional MAPKs include the p38 isoforms JNK $1 / 2 / 3$ and ERK1/2. These MAPKs are regulated by a series of upstream kinases including TAK1 and apoptosis-signal regulating kinase (ASK) (15). The ASK family of MAP3Ks (ASK1, ASK2, and ASK3, encoded by MAP3K5, MAP3K6, and MAP3K15, respectively) is related by sequence homology and its members interact to regulate the activity of one another (1618). ASK1 is the most studied family member and is an upstream kinase of the JNK and p38 pathways (19). Diverse stimuli such as TLR signaling, ROS, and ER stress can lead to ASK1 activation. Although ASK1 has been shown to regulate cell death by apoptosis (20) and ferroptosis (21), its role in myeloproliferative disorders and inflammation is not yet established.

Here, we show that ASK signaling plays a critical role in driving Ptpn $6^{\text {spin }}$-mediated inflammation by regulating the hallmark inflammatory cytokines. Ablation of Ask1 and Ask2 in Ptpn $6^{\text {spin }}$ mice significantly $(P<0.0001)$ rescues the cutaneous inflamma- 


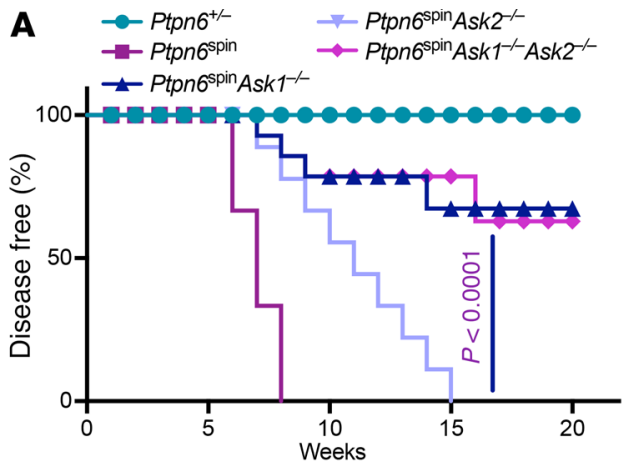

C
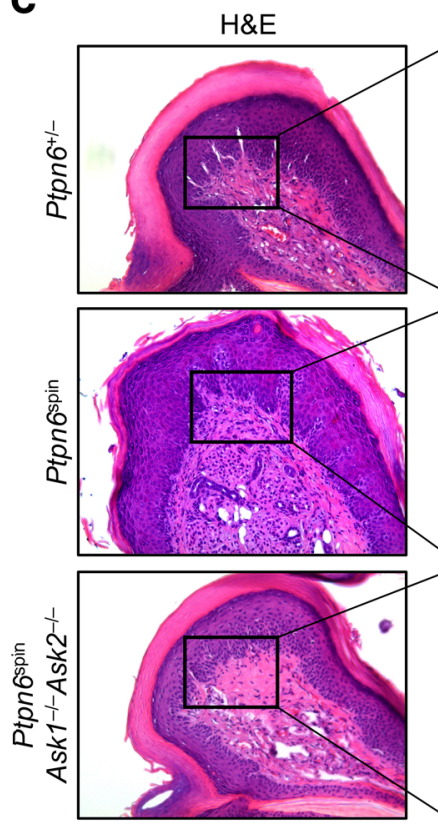
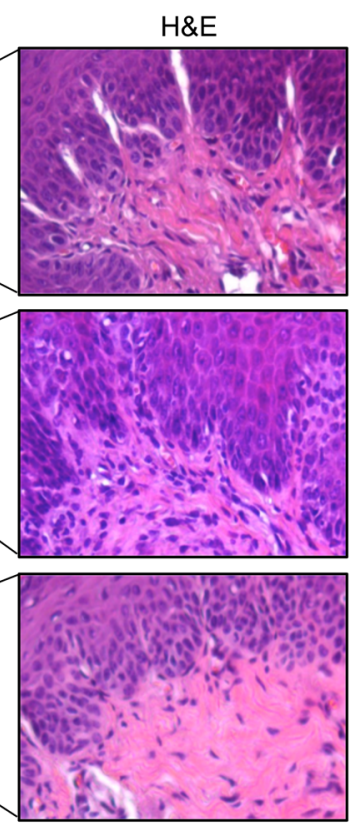

B

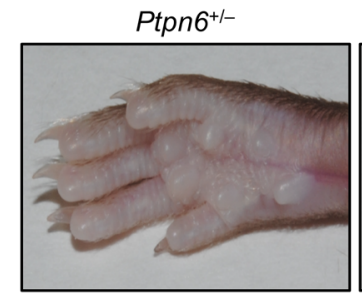

Ptpn6spin Ask ${ }^{-1-}{ }^{-}$skk2 ${ }^{-1}$

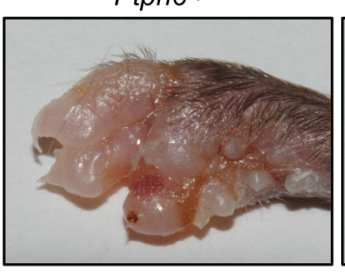

D

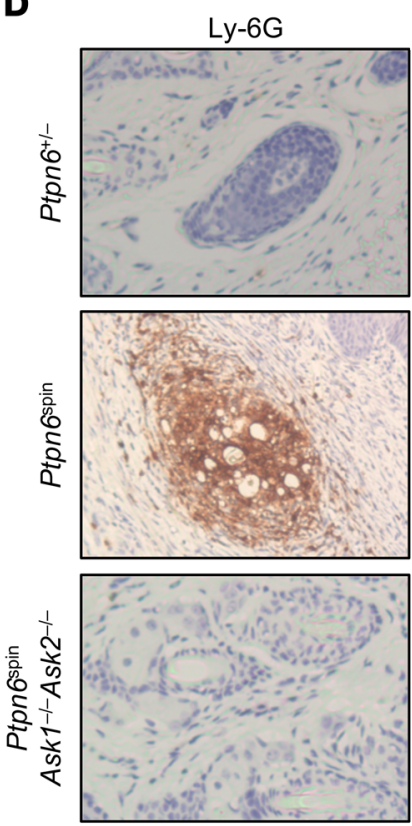

$\mathbf{E}$

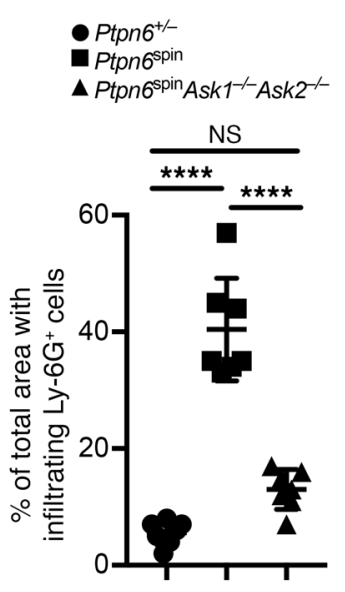

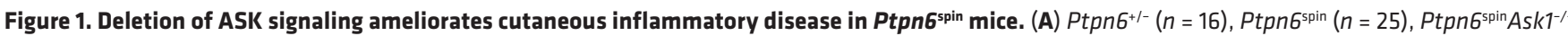
$(n=12), \operatorname{Ptpn}^{\text {spin }} A s k 2^{-/-}(n=9)$, and Ptpn6 spin Ask1 $1^{-/-} A s k 2^{-/-}(n=28)$ crosses were observed for disease progression. (B) Footpad images, (C) H\&E staining, and (D) immunohistochemistry staining of neutrophils (Ly-6G) of Ptpn6 $6^{+/-}$, Ptpn6 ${ }^{\text {spin }}$, and Ptpn6 $6^{\text {spin }} A s k 1^{-/-}$Ask2 ${ }^{-/-}$mice (original magnification, $\left.\times 20\right)$. (E) Quantification of the percentage of total area with infiltrating Ly-6C+ cells shown in $\mathbf{D}$. Disease curves in A were analyzed by Mantel-Cox log-rank test. (E) Two-way ANOVA was used to determine the significance between the 2 groups analyzed. NS, not significant. **** $P<0.0001$.

tory disease. ASK1/2 signaling plays a pivotal role in regulating Ptpn $6^{\text {spin }}$-mediated disease through the control of NF- $\kappa$ B, ERK, and p38 downstream of the IL-1R and TLR pathways, thereby controlling production of proinflammatory cytokines. Taken together, these data show that in addition to its well-known role in apoptosis, the ASK1/2 signaling complex plays a major role in

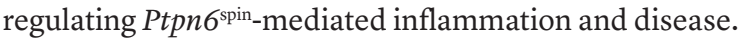

\section{Results and Discussion}

Deletion of ASK signaling ameliorates cutaneous inflammatory disease in Ptpn $6^{\text {spin }}$ mice. The role of IL-1 receptor- and MyD88-driven signaling has been well established in provoking inflammation and inflammatory skin disease in Ptpn $6^{\text {spin }}$ mice $(3,5)$. Several studies have demonstrated TAK1 as an essential central regulator of NF- $\kappa \mathrm{B}, \mathrm{ERK}$, and p38 downstream of TLR activation $(11,12)$. We hypothesized that other MAPKs (ASK1 and ASK2) can also play a crucial role in mediating the inflammatory skin disease in $P \operatorname{tpn} 6^{\text {spin }}$ mice. To identify the new players involved in this cutane- ous inflammation, we generated $P \operatorname{tpn} 6^{\text {spin }} A s k 1^{-/}, P t p n 6^{\text {spin }} A s k 2^{-/}$, and $P$ tpn $6^{\text {spin }}$ Ask1 $^{-/-}$Ask2 $2^{-/-}$mice. Ptpn6 $6^{\text {spin/+ }}$ (denoted as Ptpn6 $6^{+/-}$ hereafter) heterozygous mice did not develop any signs of footpad inflammation and were used as controls. Consistent with the previous findings $(3,7)$, Ptpn $6^{\text {spin }}$ mice spontaneously developed footpad inflammation between 6-10 weeks. Interestingly, Ptpn ${ }^{\text {spin }} A s k 1^{-/}$and Ptpn $6^{\text {spin }} A s k 1^{-/-} A s k 2^{-/-}$mice demonstrated significantly delayed disease progression as compared with $P \operatorname{tpn} 6^{\text {spin }}$ mice, and approximately $60 \%$ of the Ptpn $6^{\text {spin }} A s k 1^{-/-}$and

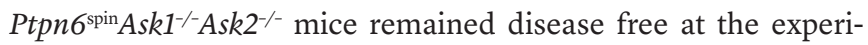
mental end point of 20 weeks with no evident signs of footpad swelling (Figure 1A). On the other hand, Ptpn $6^{\text {spin }} A s k 2^{-/-}$mice failed to provide any significant protection from the disease (Figure 1A), suggesting that deletion of ASK1 alone is sufficient to provide significant protection in $P \operatorname{tpn} 6^{\text {spin }}$ mice. However, it should be noted that the progression of disease was delayed in Ptpn $6^{\mathrm{spin}} A s k 2^{-/-}$mice compared with $P$ tpn $\sigma^{\text {spin }}$ mice, suggesting a partial protective role for ASK2. Histological analysis confirmed that these mice 


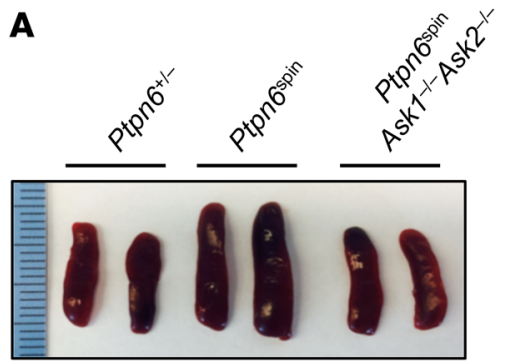

$\mathbf{E}$

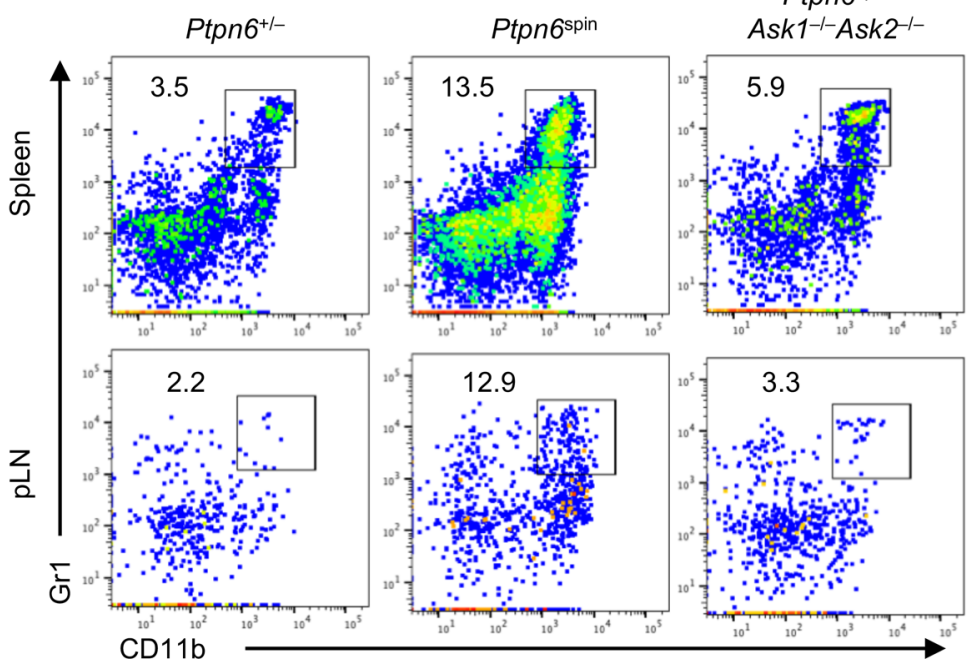

B

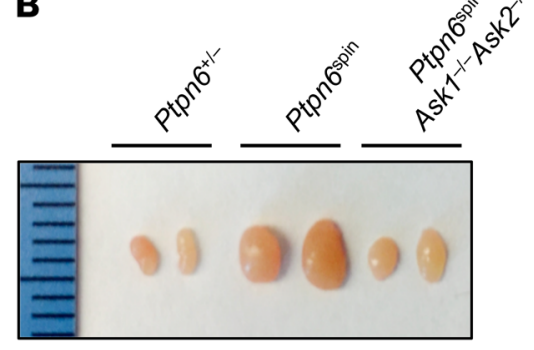

Ptpn6 6 spin

$$
\text { . }
$$

C

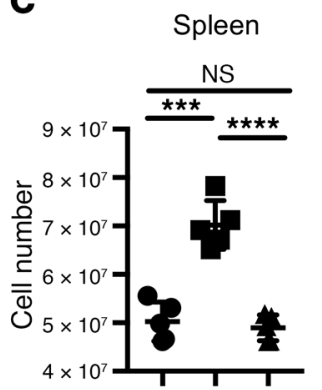

$P_{t p n} 6^{+/-}$

Ptpn6spir

A Ptpn6 ${ }^{\text {spin }}$ Ask $1^{-1-}$ Ask2 ${ }^{-1}$

$\mathbf{F}$

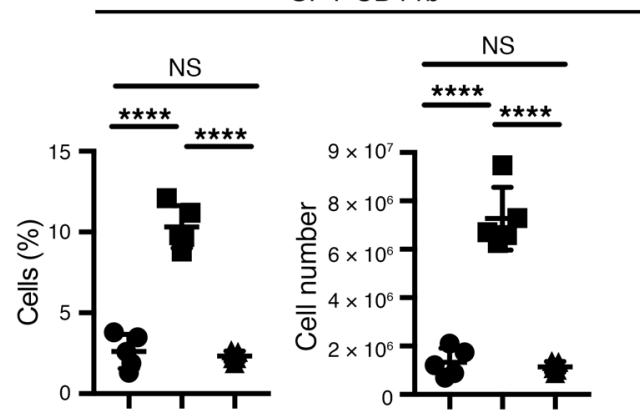

Figure 2. ASK1 and ASK2 deletion attenuates massive infiltration of myeloid cells in Ptpn6 ${ }^{\text {spin }}$ mice. Representative size of spleen (A) and popliteal

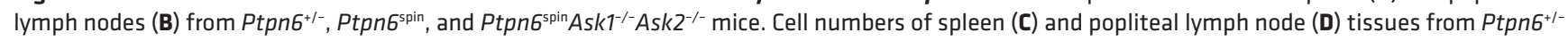
$(n=7), \operatorname{Ptpn}^{\text {spin }}(n=12)$, and Ptpn ${ }^{\text {spin }} A s k 1^{-1-} A s k 2^{-/-}(n=9)$ mice. (E) Flow cytometry analysis of Gr-1+CD11 $\mathrm{b}^{+}$neutrophil population in spleen and popliteal

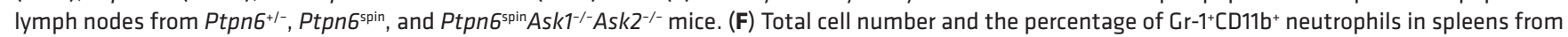
Ptpn6 $6^{+-}, P \operatorname{tpn} 6^{\text {spin }}$, and $P \operatorname{tpn} 6^{\text {spin }} A s k 1^{-/-}$Ask2 $2^{---}$mice. Each point represents an individual mouse, and the line represents mean \pm SEM (C, D, and F). Two-way ANOVA was used to determine significance between the 2 groups analyzed. NS, not significant. ${ }^{* *} P<0.01,{ }^{* *} P<0.001,{ }^{* * *} P<0.0001$.

showed much less inflammation and fewer lesions compared with Ptpn6 $^{\text {spin }}$ mice (Figure 1, B and C). Since the inflammation is characterized by heavy infiltration of neutrophils, we further analyzed the footpad sections by neutrophil-specific immunostaining. The staining showed severe neutrophilia in $P \operatorname{tpn} 6^{\text {spin }}$ mice, which was completely rescued in the Ptpn $6^{\text {spin }} A s k 1^{-/-} A s k 2^{-/-}$mice (Figure 1, D and E). These findings establish ASK as a critical kinase driving neutrophilic inflammation in Ptpn $6^{\text {spin }}$ mice.

ASK1 and ASK2 deletion attenuates massive infiltration of myeloid cells in Ptpn $6^{\text {spin }}$ mice. The neutrophilic infiltration and footpad swelling are often characterized by enlargement of popliteal lymph nodes that drain the inflamed feet. We examined Ptpn $6^{+/}$, Ptpn6 $6^{\text {spin }}$, and Ptpn6 ${ }^{\text {spin }} A s k 1^{-/-} A s k 2^{-/-}$mice to confirm the presence of any such abnormalities. Consistent with previous reports (3, 5), Ptpn $^{\text {spin }}$ mice displayed splenomegaly in addition to lymphomegaly in the popliteal lymph nodes that drain the inflamed feet (Figure 2, A and B). The enlarged spleen and popliteal lymph nodes observed in Ptpn $^{\text {spin }}$ mice were completely reversed in Ptpn 6 $6^{\text {spin }} A s k 1^{-/-} A s k 2^{-/}$mice. In concurrence with the rescue of spleen and popliteal lymph node sizes, the cell numbers in the spleen and popliteal lymph nodes of $P \operatorname{tpn} 6^{\text {spin }} A s k 1^{-/-} A s k 2^{-/-}$mice were significantly lower than the cell numbers in Ptpn $^{\text {spin }}$ mice (Figure 2, C and D). Flow cytometric analysis further revealed that the percentages of $\mathrm{Gr}^{+} \mathrm{CD}_{11} \mathrm{~b}^{+}$neutrophils in the spleen and popliteal lymph nodes of $P t p n 6^{\text {spin }} A s k 1^{-/-} A s k 2^{-/-}$mutant mice tend to be comparable to those in $P$ tpn $\sigma^{+/-}$heterozygous mice and are significantly lower than those in Ptpn ${ }^{\text {spin }}$ mice (Figure 2, E and $\mathrm{F}$ ), indicating that the increased numbers of infiltrating and circulating myeloid cells are responsible for causing the suppurative inflammation in the footpads of Ptpn $\sigma^{\text {spin }}$ mice.

ASK1/2 instigates Ptpn $6^{\text {sin }}$-mediated disease by promoting proinflammatory signaling. Myeloid cells, specifically neutrophils, are hyperactivated in $P t p n \sigma^{\text {spin }}$ mice and produce increased amounts of proinflammatory cytokines such as granulocyte CSF (G-CSF), CXCL1 chemokine KC (CXCL1/KC), and IL-6 $(5,9)$. We measured the levels of these hallmark cytokines involved in instigating the Ptpn $6^{\text {spin }}$-associated inflammatory disease progression and neutrophilia. Whereas the serum concentrations of G-CSF, IL-6, CXCL1/KC, MCP-1, IL-1 $\alpha$, and TNF in Ptpn $6^{\text {spin }}$ mice were highly increased, the levels of these cytokines and chemokines were significantly reduced in the sera from Ptpn ${ }^{\mathrm{spin}} A s k 1^{-/-} A s k 2^{-\gamma_{-}}$mutant mice (Figure 3A and Supplemental Figure 1A; supplemental material available online with this article; https://doi.org/10.1172/ JCI98446DS1), indicating an increase in the number of circulating neutrophils in the peripheral blood. The obvious visible defect is in the footpads of the Ptpn $6^{\text {spin }}$ mice, so we first analyzed mRNA 
A
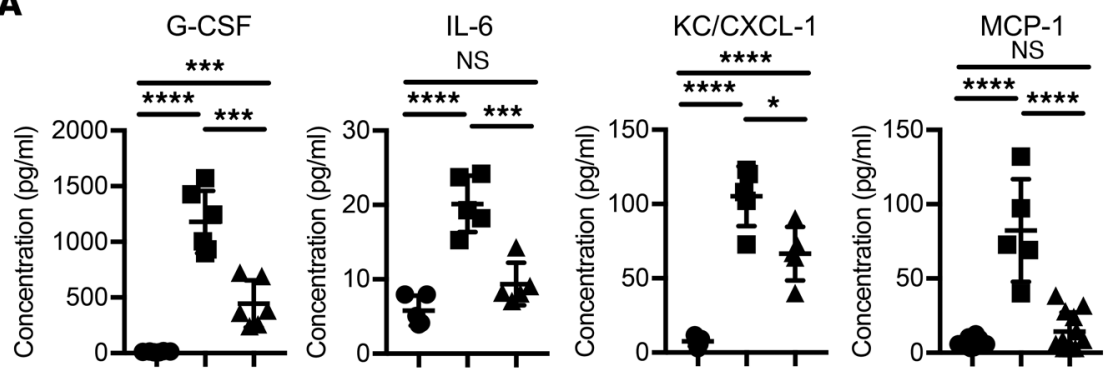

- Ptpn6 $^{+/}$

- Ptpn6 6 spin

- Ptpn6 ${ }^{\text {spin }}$ Ask $1^{-1-}$ Ask2 $2^{-1}$

B
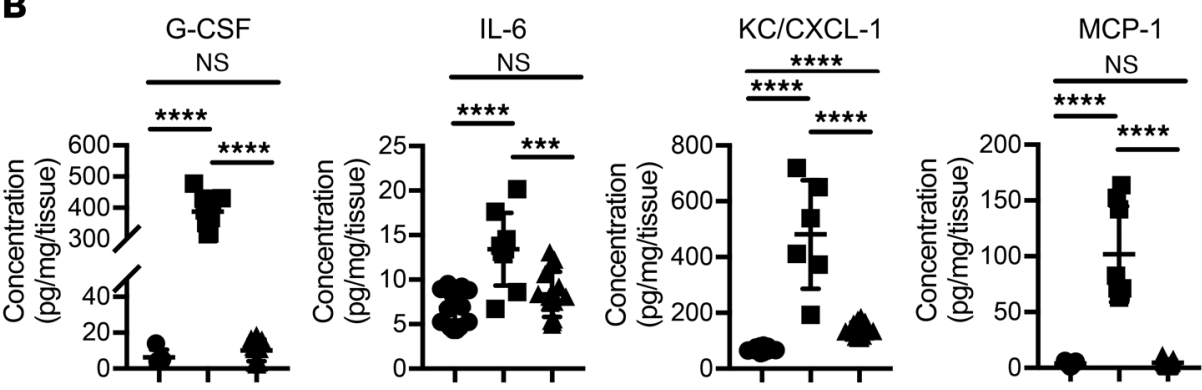

- Ptpn $^{+/}$

- Ptpn6 6 spin

- Ptpn6 ${ }^{\text {spin }}$ Ask $1^{-1-}$ Ask2 ${ }^{--}$

C

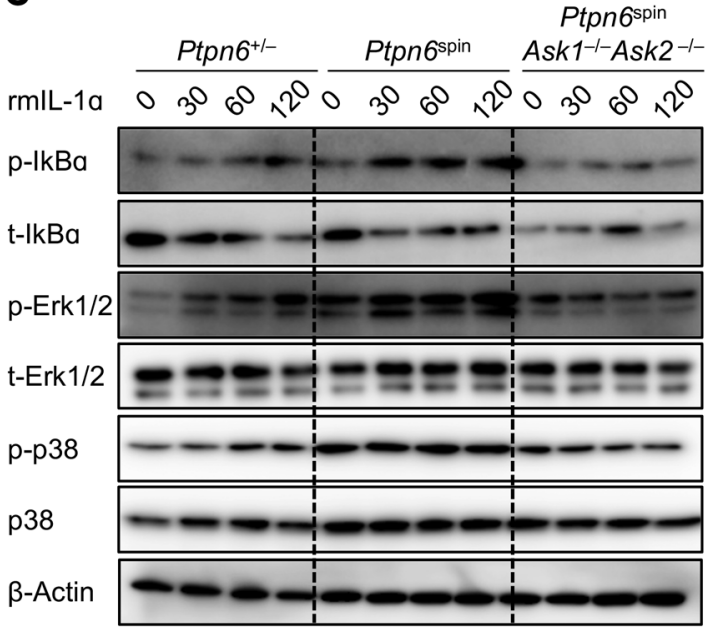

D

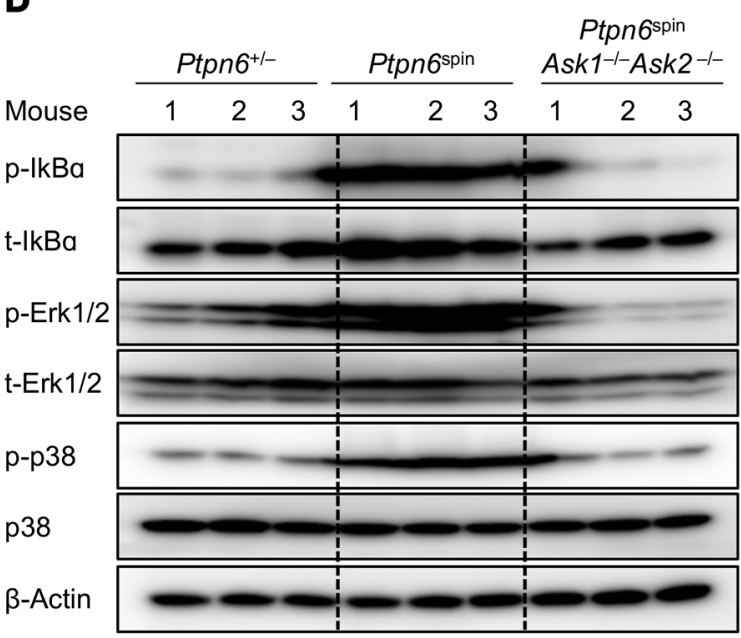

Figure 3. ASK1/2 instigates Ptpn6 $6^{\text {spin }}$-mediated disease by promoting proinflammatory signaling. (A) Serum was harvested from Ptpn6 ${ }^{+/-}(n=7)$, Ptpn $6^{\text {spin }}$ $(n=12)$, and Ptpn $6^{\text {spin }} A s k 1^{-/} A s k 2^{-/-}(n=9)$ mice and the concentrations of cytokines (G-CSF, IL-6) and chemokines (KC/CXCL-1, MCP-1) were measured by ELISA. (B) Footpads from Ptpn6 $6^{+-}$, Ptpn $6^{\text {spin }}$, and Ptpn $6^{\text {spin }} A s k 1^{-1-}$ Ask $2^{-1-}$ mice were homogenized and the concentrations of cytokines (G-CSF, IL-6) and chemokines (KC/CXCL-1, MCP-1) were measured by ELISA in the quantified lysates. All data are mean \pm SEM. Each point represents an individual mouse, and the line represents mean \pm SEM. (C) Neutrophils from bone marrow were stimulated with recombinant mouse IL-1 $\alpha$ ( $10 \mathrm{ng} / \mathrm{ml})$ for the indicated time period. Whole-cell lysates were prepared and expression of $p-I \kappa B \alpha, I \kappa B \alpha, p-E R K 1 / 2, E R K 1 / 2, p-p 38$, and $p 38$ was determined by Western blotting. (D) Footpad lysates were prepared and expression of $\mathrm{p}-\mathrm{I} \mathrm{\kappa} \mathrm{B} \alpha, \mathrm{I} \mathrm{K} \mathrm{B} \alpha, \mathrm{p}$-ERK1/2, ERK1/2, p-p38, and $\mathrm{p} 38$ was determined by Western blotting. $\beta$-Actin was used as an internal control. Results are representative of 3 independent experiments. (A and B) Two-way ANOVA was used to determine the significance between the 2 groups analyzed. NS, not significant. ${ }^{*} P<0.05,{ }^{* * *} P<0.001,{ }^{* * *} P<0.0001$.

expression of Illa and Tnf in the footpads and the draining lymph nodes of these mice. The quantitative PCR data revealed that genes encoding IL-1 $\alpha$ and TNF were highly induced in the Ptpn $6^{\text {spin }}$ mice. However, this aberrant induction of Illa and $\operatorname{Tn} f$ was restored in the Ptpn $6^{\text {spin }} \mathrm{Ask1}^{-/-} \mathrm{Ask}^{-/-}$mutant mice (Supplemental Figure 1, B and $\mathrm{C})$, indicating that IL- $1 \alpha$-driven signaling promotes autoinflammatory disease in Ptpn $6^{\text {spin }}$ mice. We next investigated the cytokine and chemokine concentrations in the footpads of these mice. Similarly, the levels of G-CSF, IL-6, CXCL1/KC, and MCP-1 were significantly reduced in the footpads of $P \operatorname{tpn} 6^{\text {spin }} A s k 1^{-/-} A s k 2^{-/-}$mutant mice when compared with levels in Ptpn $6^{\text {spin }}$ mice (Figure $3 B$ ). At the site of inflammation, an increase in cytokine surge correlates with an increase in the number of circulating neutrophils in the peripheral blood. These results indicate that ASK1 and ASK2 each play a crucial role in Ptpn $6^{\text {spin }}$-mediated inflammation by regulating the production of proinflammatory cytokines and chemokines. Given that IL- $1 \alpha$ acts as an alarmin that contributes to the inflammatory disease and wound-healing responses in Ptpn $6^{\text {spin }}$ mice (5, 9), we performed microabrasion injury experiments with WT and Ptpn ${ }^{\text {spin }} A s k 1^{-/-} A s k 2^{-/-}$prediseased mice. The microabrasion procedure triggered a rapid (5 hours after wound induction) and potent production of inflammatory cytokines and chemokines in WT mice. 
Notably, the enhanced secretion of neutrophilic factors was significantly lowered in Ptpn $6^{\text {spin }} A s k 1^{-/-} A s k 2^{-/-}$mice (Supplemental Figure 2, A-C). We further isolated the neutrophils from the $P t p n 6^{+-}$, Ptpn $6^{\text {spin }}$, and Ptpn $6^{\text {spin }} A s k 1^{-/-}$Ask2 $2^{-/}$mice and stimulated them with recombinant murine IL-1 $\alpha$ to investigate whether ASKs are involved in IL-1 $\alpha$-mediated signaling. Stimulation of neutrophils with IL-1 $\alpha$ led to increased MAPK and NF- $\mathrm{KB}$ signaling in $P \operatorname{tpn} 6^{\text {spin }}$ mice, but significantly reduced signaling in the Ptpn $6^{\text {spin }} A s k 1^{-/-} A s k 2^{---}$mice (Figure 3C). These results further demonstrate that IL-1 $\alpha$ acts as the apical cytokine that signals via ASK1/2 and instigates the inflammatory cascade in $P t p n 6^{\text {spin }}$ mice. To confirm the specificity of this IL- $1 \alpha$-mediated signaling, we further analyzed the involvement of interferon signaling. Upon stimulation of neutrophils from $P t p n 6^{+/-}$, Ptpn $6^{\text {spin }}$, and Ptpn $6^{\text {spin }} A s k 1^{-/-} A s k 2^{-/-}$mice with recombinant IFN- $\beta$, we did not observe any difference in the STAT signaling among Ptpn 6 ${ }^{+/}$, Ptpn $^{\text {spin }}$, and Ptpn $6^{\text {spin }} A s k 1^{-/-} A s k 2^{-/-}$KO neutrophils (Supplemental Figure 3). These data are in line with a recent study showing that knockout of IFNAR did not provide protection in Ptpn $6^{\text {spin }}$ mice (9). Taken together, these data suggest that inflammatory disease in Ptpn $6^{\text {spin }}$ mice is independent of interferon signaling. Since ASK1 has been shown to be involved in regulating activation of JNK and p38 downstream of TLRs (19), we hypothesized that targeted MAPK and NF- $\mathrm{KB}$ signaling drives Ptpn $6^{\text {spin }}$-associated inflammation. We found that in vivo ASK1 and ASK2 deletion markedly reduced local activation of MAPK and NF- $\mathrm{BB}$ signaling in the footpads (Figure 3D). Taken together, these results are consistent with the hypothesis that IL-1 $\alpha$ acts as an apical cytokine that promotes ASK1/2-mediated proinflammatory cytokine and chemokine responses, which ultimately recruit myeloid cells to drive autoinflammatory skin disease in $P$ tpn $6^{\text {spin }}$ mice.

To address the clinical significance of IL- $1 \alpha$ and ASK signaling in humans, we performed the knockdown of ASK1 and ASK2 in HEK293T cells (Supplemental Figure 4, A-C). After confirming the successful knockdown, we stimulated the cells with IL-1 $\alpha$ and checked for MAPK signaling. Although IL-1 $\alpha$ stimulation did induce MAPK signaling in the control cells, the levels of induction were reduced in the cells with single knockdown of ASK1 and double knockdown of ASK1 and ASK2 (Supplemental Figure 4, D-F). Interestingly, single knockdown of ASK2 did not show any difference in the activation of MAPK signaling. Consistent with the mice data presented here, double knockdown of ASK1 and ASK2 showed impaired MAPK signaling in response to IL-1 $\alpha$ compared with the control cells.

Our results highlight a critical role for ASK-mediated signaling in myeloid cells in driving an inflammatory circuit that triggers excessive inflammatory responses and persistent tissue damage in a mouse model of neutrophilic dermatosis. We have demonstrated that ASK signaling plays an important role in instigating inflammatory disease in Ptpn $6^{\text {spin }}$ mice, as its absence resulted in a substantial resolution of inflammation. The lack of ASK1 and ASK2 in Ptpn $6^{\text {spin }}$ neutrophils led to a marked downregulation of ERK and NF- $\mathrm{B}$ signalingcascades, resultingina dramaticdecreaseininflammatory cytokine production. In summary, our study defines a role for ASK1 and ASK2 in promoting inflammation and disease. Consequently, therapeutic inhibition of ASK1 and ASK2 activity may provide novel approaches to break the self-reinforcing inflammatory circuits that drive chronic autoinflammatory and autoimmune diseases.

\section{Methods}

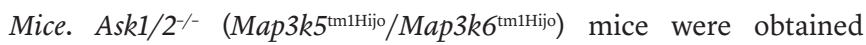
from the RIKEN BioResources Center and Ptpn $6^{\text {spin }}$ mice have been described previously (3). Ptpn $6^{\text {spin }} A s k 1^{-/} A s k 2^{-/}, P t p n 6^{\text {spin }} A s k 1^{-/}$, and $P \operatorname{tpn} 6^{\text {spin }} A s k 2^{--}$mice were generated by crossing $P$ tpn $6^{\text {spin }}$ mice with

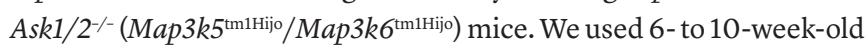
male and female mice (littermates) in this study unless stated otherwise. All mice were kept in specific pathogen-free conditions within the Animal Resource Center at St. Jude Children's Research Hospital.

Neutrophil isolation and in vitro stimulation. Bone marrow cells were isolated from the femurs of mice and neutrophils $\left(\mathrm{CD} 11 \mathrm{~b}^{+} \mathrm{Gr}-1^{+}\right)$ were purified by FACS as described elsewhere (5). Neutrophils $\left(1 \times 10^{6}\right.$ cells $/ \mathrm{ml}$ ) were stimulated with $10 \mathrm{ng} / \mathrm{ml}$ recombinant murine IL- $1 \alpha$ (Gold Biotechnology) or $100 \mathrm{U} / \mathrm{ml}$ recombinant IFN- $\beta$ (PBL Biosciences) for indicated time periods. Cell lysates were collected in RIPA lysis buffer and immunoblotting was performed.

Histopathology. Formalin-preserved feet were processed and embedded in paraffin according to standard procedures. Sections $(5 \mu \mathrm{m})$ were stained with hematoxylin and eosin (H\&E). For immunohistochemistry, formalin-fixed, paraffin-embedded tissues were cut into $4-\mu \mathrm{m}$ sections and slides were stained with anti-Ly-6G to highlight neutrophils in the footpads, and the images were acquired using light microscopy.

Immunoblot analysis. Footpad protein lysates were collected in RIPA lysis buffer supplemented with complete protease inhibitor cocktail (Roche) and PhosSTOP (Roche) using a tissue homogenizer. Samples were quantified using a Pierce BCA Protein Assay Kit per the manufacturer's instructions, and $40 \mu \mathrm{g}$ protein was resolved by SDS-PAGE and transferred to PVDF membranes. Membranes were blocked in $5 \%$ nonfat milk and incubated overnight at $4^{\circ} \mathrm{C}$ with primary antibodies. The membranes were then probed with HRPtagged secondary antibodies at room temperature for 1 hour. Immunoreactive proteins were visualized using the Luminata Western HRP chemiluminescence substrate. Antibodies against the following proteins were obtained from Cell Signaling Technology: phosphoERK1/2 (catalog 9101), ERK1 (catalog 9102), phospho-p38 (catalog 9211), p38 (catalog 9212), phospho-IкB $\alpha$ (catalog 2859), IкB $\alpha$ (cata$\log$ 9242), phospho-STAT1 (catalog 9167). Actin was obtained from Proteintech (catalog 66009-1-IG).

In vivo cytokine levels. Blood was collected by submandibular venipuncture and allowed to clot for 60 minutes at room temperature. Serum was collected after centrifugation and cytokines were measured by ELISA. Footpad protein lysates were processed as described above and cytokines were measured by ELISA.

ELISA. Cytokine ELISAs were performed according to the manufacturer's instructions (Milliplex).

Flow cytometry. CD11b (M1/70; Invitrogen) and Gr-1 (RB6-8C5; Biolegend) antibodies were used for flow cytometry. Flow cytometry data were acquired on LSR Fortessa (BD Biosciences) and analyzed using Flowjo software (Tree Star).

RNA interference. For RNAi, HEK293T cells (CRL-3216, ATCC) were transfected with the following siRNAs using Lipofectamine RNAi MAX (Invitrogen) according to the manufacturer's instructions: validated human ASK1 (siRNA1, Stealth Select RNAi MAP3K5VHS40812) and validated human ASK2 (siRNA 2, Stealth Select RNAi MAP3K6VHS40537).

Statistics. All results are presented as mean \pm SEM. Disease curves were analyzed by performing Mantel-Cox log-rank testing, and significant differences between 2 groups were determined using the Mann- 
Whitney $U$ test. Statistical analysis among samples was performed using the 2-tailed Student's $t$ test and 2-way ANOVA for multiple comparisons. All the analysis was done using Graphpad Prism software (version 7.0). Differences were considered statistically significant when $P<0.05$. NS, not significant. ${ }^{*} P<0.05,{ }^{* *} P<0.01,{ }^{* *} P<0.001$, ${ }^{* * * *} P<0.0001$.

Study approval. All the animal studies were approved by the Institutional Animal Care and Use Committee of St. Jude Children's Research Hospital, Memphis, TN.

\section{Author contributions}

ST, PG, and TDK designed and conceptualized the study. ST, $\mathrm{AB}, \mathrm{TKD}$, and $\mathrm{PG}$ performed the experiments. ST analyzed the data and wrote the manuscript with input from other authors. TDK oversaw the project.

\section{Acknowledgments}

This work was supported by grants to TDK from the NIH (CA163507, AR056296, AI124346, and AI101935) and the American Lebanese Syrian Associated Charities (ALSAC).

Address correspondence to: Thirumala-Devi Kanneganti, Department of Immunology, St. Jude Children's Research Hospital, MS \#351, 570, St. Jude Place, Suite E7004, Memphis, Tennessee 38105-2794, USA. Phone: 901.595.3634; Email: thirumaladevi.kanneganti@stjude.org.
1. Cao H, Hegele RA. Identification of polymorphisms in the human SHP1 gene. J Hum Genet. 2002;47(8):445-447.

2. Christophi GP, et al. SHP-1 deficiency and increased inflammatory gene expression in PBMCs of multiple sclerosis patients. Lab Invest. 2008;88(3):243-255.

3. Croker BA, et al. Inflammation and autoimmunity caused by a SHP1 mutation depend on IL-1, MyD88, and a microbial trigger. Proc Natl Acad Sci US A. 2008;105(39):15028-15033.

4. Nesterovitch AB, et al. Alteration in the gene encoding protein tyrosine phosphatase nonreceptor type 6 (PTPN6/SHP1) may contribute to neutrophilic dermatoses. Am J Pathol. 2011;178(4):1434-1441.

5. Lukens JR, et al. RIP1-driven autoinflammation targets IL-1 $\alpha$ independently of inflammasomes and RIP3. Nature. 2013;498(7453):224-227.

6. Lear JT, Atherton MT, Byrne JP. Neutrophilic dermatoses: pyoderma gangrenosum and Sweet's syndrome. Postgrad Med J. 1997;73(856):65-68.

7. Lukens JR, Kanneganti TD. SHP-1 and IL-1 $\alpha$ conspire to provoke neutrophilic dermatoses. Rare Dis. 2014;2:e27742.

8. Abram CL, Roberge GL, Pao LI, Neel BG, Lowell
CA. Distinct roles for neutrophils and dendritic cells in inflammation and autoimmunity in motheaten mice. Immunity. 2013;38(3):489-501.

9. Gurung P, Fan G, Lukens JR, Vogel P, Tonks NK, Kanneganti TD. Tyrosine kinase SYK licenses MyD88 adaptor protein to instigate IL-1 $\alpha$ mediated inflammatory disease. Immunity. 2017;46(4):635-648.

10. Gurung P, Kanneganti TD. Autoinflammatory skin disorders: the inflammasomme in focus. Trends Mol Med. 2016;22(7):545-564.

11. Sato S, et al. Essential function for the kinase TAK1 in innate and adaptive immune responses. Nat Immunol. 2005;6(11):1087-1095.

12. Shim JH, et al. TAK1 is an essential regulator of BMP signalling in cartilage. EMBO J. 2009;28(14):2028-2041.

13. Ajibade AA, et al. TAK1 negatively regulates NF- $\kappa$ B and p38 MAP kinase activation in Gr-1+CD11b+ neutrophils. Immunity. 2012;36(1):43-54.

14. Cargnello M, Roux PP. Activation and function of the MAPKs and their substrates, the MAPKactivated protein kinases. Microbiol Mol Biol Rev. 2011;75(1):50-83.

15. Arthur JS, Ley SC. Mitogen-activated protein kinases in innate immunity. Nat Rev Immunol.
2013;13(9):679-692.

16. Kaji T, et al. ASK3, a novel member of the apoptosis signal-regulating kinase family, is essential for stress-induced cell death in HeLa cells. Biochem Biophys Res Commun. 2010;395(2):213-218.

17. Federspiel JD, et al. Assembly dynamics and stoichiometry of the apoptosis signal-regulating kinase (ASK) signalosome in response to electrophile stress. Mol Cell Proteomics. 2016;15(6):1947-1961.

18. Takeda K, et al. Apoptosis signal-regulating kinase (ASK) 2 functions as a mitogenactivated protein kinase kinase kinase in a heteromeric complex with ASK1. JBiol Chem. 2007;282(10):7522-7531.

19. Tobiume K, et al. ASK1 is required for sustained activations of JNK/p38 MAP kinases and apoptosis. EMBO Rep. 2001;2(3):222-228.

20. Fujino G, et al. Thioredoxin and TRAF family proteins regulate reactive oxygen species-dependent activation of ASK1 through reciprocal modulation of the N-terminal homophilic interaction of ASK1. Mol Cell Biol. 2007;27(23):8152-8163.

21. Hattori K, Ishikawa H, Sakauchi C, Takayanagi S, Naguro I, Ichijo H. Cold stress-induced ferroptosis involves the ASK1-p38 pathway. EMBO Rep. 2017;18(11):2067-2078. 